CRYSTALLOGRAPHIC COMMUNICATIONS

ISSN 2056-9890

Received 12 June 2017

Accepted 19 June 2017

Edited by M. Weil, Vienna University of Technology, Austria

Keywords: crystal structure; redetermination; mercury; thiocyanate.

CCDC reference: 1556957

Supporting information: this article has supporting information at journals.iucr.org/e

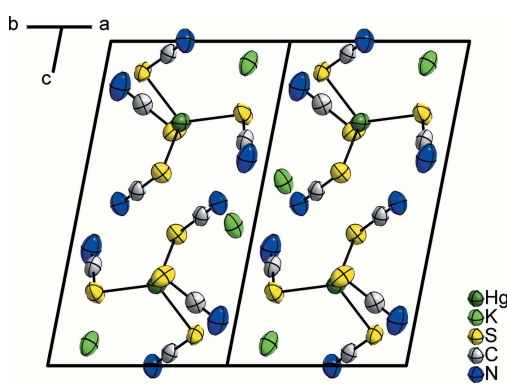

OPEN $\odot$ ACCESS

\section{Redetermination of the crystal structure of $\mathrm{K}_{2} \mathrm{Hg}(\mathrm{SCN})_{4}$}

\author{
Jascha Bandemehr, Matthias Conrad and Florian Kraus*
}

Anorganische Chemie, Fachbereich Chemie, Philipps-Universität Marburg, Hans-Meerwein-Strasse 4, 35032 Marburg. *Correspondence e-mail: florian.kraus@chemie.uni-marburg.de

Single crystals of $\mathrm{K}_{2} \mathrm{Hg}(\mathrm{SCN})_{4}$ [dipotassium tetrathiocyanatomercurate(II)] were grown from aqueous solutions of potassium thiocyanate and mercury(II) thiocyanate and studied by single-crystal X-ray diffraction. In comparison with the previously reported structure model [Zvonkova (1952). Zh. Fiz. Khim. 26, 1798-1803], all atoms in the crystal structure were located, with lattice parameters and fractional coordinates determined to a much higher precision. In the (crystal) structure, the $\mathrm{Hg}^{\mathrm{II}}$ atom is located on a twofold rotation axis and is coordinated in the form of a distorted tetrahedron by four $\mathrm{S}$ atoms of the thiocyanate anions. The $\mathrm{K}^{+}$cation shows a coordination number of eight.

\section{Chemical context}

In search for suitable educts for fluorination we thought that $\mathrm{K}_{2} \mathrm{Hg}(\mathrm{SCN})_{4}$ would be a well-suited candidate. Once we had obtained the compound, we noticed that the original structure determination (Zvonkova, 1952) was of low precision with the light atoms ( $\mathrm{C}$ and $\mathrm{N}$ ) not determined, so we redetermined the crystal structure to much higher precision and accuracy.

$\mathrm{K}_{2} \mathrm{Hg}(\mathrm{SCN})_{4}$ was first synthesized in 1901 (Rosenheim \& Cohn, 1901) by adding an aqueous solution of potassium thiocyanate to a boiling solution of mercury(II) thiocyanate and crystallization upon cooling to room temperature. The crystal structure has been known since 1952 (Zvonkova, 1952) and IR spectra were first measured in 1962 (Tramer, 1962). Related compounds of the type $A_{2} \mathrm{Hg}(\mathrm{SCN})_{4}$ with $A=\mathrm{Rb}, \mathrm{Cs}$, $\mathrm{NH}_{4}, \mathrm{NMe}_{4}$ are also known (Larbot \& Beauchamp, 1973; Tramer, 1962). The $\mathrm{Hg}^{\mathrm{II}}$ atom in $\mathrm{K}_{2} \mathrm{Hg}(\mathrm{SCN})_{4}$ is coordinated in the form of a distorted tetrahedron by four $\mathrm{S}$ atoms in a fashion similar to the $\mathrm{Hg}^{\mathrm{II}}$ atom in the structure of $\mathrm{CoHg}(\mathrm{SCN})_{4}$ (Jefferey \& Rose, 1968). Such tetrahedrally coordinated $\mathrm{Hg}^{\mathrm{II}}$ atoms are also known, for example, for the halide and pseudo-halide compounds $A_{2} \mathrm{Hg} X_{4}$, viz. $\mathrm{Cs}_{2} \mathrm{HgBr}_{4}$ (Pakhomov et al., 1978; Altermatt et al., 1984; Pinheiro et al., 1998), $\mathrm{Cs}_{2} \mathrm{HgCl}_{4}$ (Linde et al., 1983; Pakhomov et al. 1992a,b; Bagautdinov \& Brown, 2000), $\mathrm{Cs}_{2} \mathrm{HgI}_{4}$ (Zandbergen et al., 1979; Pakhomov \& Fedorov, 1973), $\mathrm{K}_{2} \mathrm{Hg}(\mathrm{CN})_{4}$ (Gerlach \& Powell, 1986; Dickinson, 1922) and $\mathrm{Rb}_{2} \mathrm{Hg}(\mathrm{CN})_{4}$ (Klüfers et al., 1981).

\section{Structural commentary}

The lattice parameters obtained by our room-temperature single-crystal structure determination (Table 1) agree with those obtained previously $(a=11.04, b=9.22, c=13.18 \AA$, $\beta=$ $106.30^{\circ}, Z=4$; Zvonkova, 1952$) . \mathrm{K}_{2} \mathrm{Hg}(\mathrm{SCN})_{4}$ crystallizes in 


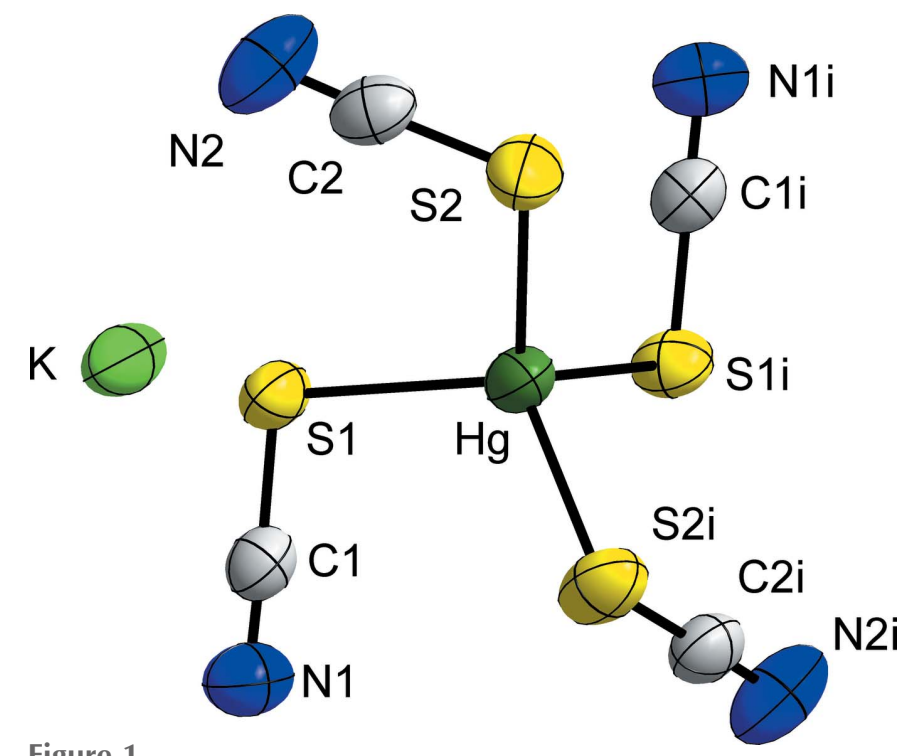

Figure 1

A section of the crystal structure of $\mathrm{K}_{2} \mathrm{Hg}(\mathrm{SCN})_{4}$, showing the $\left[\mathrm{Hg}(\mathrm{SCN})_{4}\right]^{2-}$ anion and the $\mathrm{K}^{+}$cation. Displacement ellipsoids are shown at the $70 \%$ probability level at $293 \mathrm{~K}$. [Symmetry code: (i) $-x, y$, $\left.\frac{1}{2}-z\right]$.

the monoclinic crystal system in space group C2/c (No. 15). The $\mathrm{Hg}^{\mathrm{II}}$ atom is located on a twofold rotation axis (Wyckoff position 4e) and is coordinated in the form of a distorted tetrahedron by four $\mathrm{S}$ atoms of the thiocyanate anions (Fig. 1). The $\mathrm{S}-\mathrm{Hg}-\mathrm{S}$ angles are in the range $105.02(2)-114.67(3)^{\circ}$ and the $\mathrm{Hg}-\mathrm{S}$ distances are $2.5380(8)$ and 2.5550 (7) $\AA$, both in good agreement with the previously reported data $\left(\mathrm{S}-\mathrm{Hg}-\mathrm{S}\right.$ angle: $102-118^{\circ}, \mathrm{Hg}-\mathrm{S}$ distance: 2.54 (2); Zvonkova, 1952). The $\mathrm{Hg}-\mathrm{S}$ distance is slightly longer than those of the sixfold-coordinated $\mathrm{Hg}^{\mathrm{II}}$ atom in $\mathrm{Hg}(\mathrm{SCN})_{2}$ [2.381 (6) $\AA$ ] (Beauchamp \& Goutier, 1972) and lies within the range of $\mathrm{Hg}-\mathrm{S}$ distances [2.3954 (11)$2.7653(6) \AA]$ for the threefold coordinated $\mathrm{Hg}^{\mathrm{II}}$ atom in $\mathrm{KHg}(\mathrm{SCN})_{3}$ (Weil \& Häusler, 2014).

As may be expected, the two unique $\mathrm{SCN}^{-}$anions are almost linear $\left[178.0(3), 178.2(3)^{\circ}\right]$, and the angles are comparable with those reported for $\mathrm{Hg}(\mathrm{SCN})_{2}$ [177.5 (13) ${ }^{\circ}$; Beauchamp \& Goutier, 1972] or $\mathrm{KHg}(\mathrm{SCN})_{3}$ [176.41 (4)$179.8(3)^{\circ}$; Weil \& Häusler, 2014]. The S-C [1.656 (3), 1.665 (3) $\AA$ ] and $\mathrm{C}-\mathrm{N}[1.153$ (5), 1.152 (4) $\AA$ ] distances are comparable as well [S-C: $1.62(2), \mathrm{C}-\mathrm{N}$ : 1.18 (3) $\mathrm{A}]$ (Beauchamp \& Goutier, 1972) [S-C: 1.657 (4)-1.675 (3) A, C-N: 1.140 (4)-1.145 (5) A] (Weil \& Häusler, 2014). The Hg-S-C angles in the title salt are $98.59(10)$ and $97.06(10)^{\circ}$, respectively. In comparison with the coordination polyhedron of the $\mathrm{Hg}^{\mathrm{II}}$ atom and the structural feature of the $\mathrm{SCN}^{-}$anions in $\mathrm{CoHg}(\mathrm{SCN})_{4}[\mathrm{Hg}-\mathrm{S}: \quad 2.558-2.614 \AA$ A, S-C: $1.635-1.720 \AA$, C-N: 1.200-1.322 Å, S-Hg-S angles: 105.1 (1), $108.7(1)^{\circ}$, $\mathrm{Hg}-\mathrm{S}-\mathrm{C}$ angle: $97.3(5)^{\circ}$ ] (Jefferey \& Rose, 1968), the respective angles and distances of the complex $\left[\mathrm{Hg}(\mathrm{SCN})_{4}\right]^{2-}$ anion presented here agree well. In total, a $\left[\mathrm{Hg}(\mathrm{SCN})_{4}\right]^{2-}$ anion is surrounded by twelve potassium atoms.

The $\mathrm{K}^{+}$cation shows a coordination number of eight, with disparate bond lengths that can be associated with a $[4+3+1]$ coordination. Four $\mathrm{K}-\mathrm{N}$ distances are in the range 2.816 (4)3.031 (5) $\AA$, three $\mathrm{K}-\mathrm{S}$ distances are in the range 3.4466 (11)3.5315 (12) $\AA$ and there is one very long $\mathrm{K}-\mathrm{N}$ distance of 3.793 (5) А. Therefore, the resulting coordination polyhedron is of an odd shape. The $\mathrm{K}^{+}$cation is coordinated in total by five $\left[\mathrm{Hg}(\mathrm{SCN})_{4}\right]^{2-}$ units, three of these in a monodentate manner (two via $\mathrm{N}$ atoms and one via the $\mathrm{S}$ atom of the thiocyanate anions) and the other two in a bidentate mode (via the $\mathrm{N}$ and $\mathrm{S}$ atoms of neighboring thiocyanate anions). Overall, a complex three-dimensional framework results. The crystal structure of the title compound is shown in Fig. 2.

\section{Synthesis and crystallization}

Potassium tetrathiocyanatomercurate(II) was synthesized by slowly adding a potassium thiocyanate solution $(2.076 \mathrm{~g}$, $21.36 \mathrm{mmol}$ in $10 \mathrm{ml} \mathrm{H}_{2} \mathrm{O}$ ) to a boiling solution of mercury(II) thiocyanate $\left(3.176 \mathrm{~g}, 10.03 \mathrm{mmol}\right.$ in $\left.10 \mathrm{ml} \mathrm{H}_{2} \mathrm{O}\right)$. After the formed mercury sulfide had been filtered off through a Büchner funnel, the solution was concentrated on a hot plate until crystallization set in. The crystallized product was collected on a Büchner funnel and the filtrate was allowed to stand at room temperature until crystals of much better quality were obtained. A selected colorless single crystal was investigated by X-ray diffraction. Mercury(II) thiocyanate was prepared as reported previously (Hermes, 1866) using mercury(II) nitrate and potasium thiocyanate and was recrystallized out of ethanol.

\section{Refinement}

Crystal data, data collection and structure refinement details are summarized in Table 1. As a starting model for the structure refinement, the atomic coordinates of the previously reported $\mathrm{K}_{2} \mathrm{Hg}(\mathrm{SCN})_{4}$ structure model were used (Zvonkova,

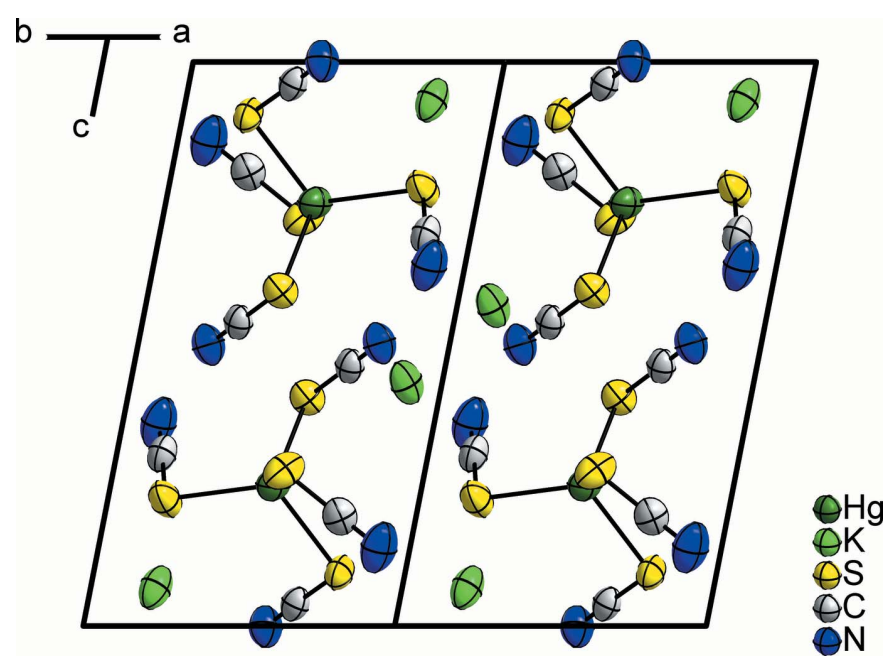

Figure 2

The crystal structure of $\mathrm{K}_{2} \mathrm{Hg}(\mathrm{SCN})_{4}$ viewed along [110]. Displacement ellipsoids are shown at the $70 \%$ probability level at $293 \mathrm{~K}$. Bonds involving the $\mathrm{K}^{+}$cation are omitted for clarity. 
Table 1

Experimental details.

\begin{tabular}{|c|c|}
\hline \multicolumn{2}{|l|}{ Crystal data } \\
\hline Chemical formula & $\mathrm{K}_{2} \mathrm{Hg}(\mathrm{SCN})_{4}$ \\
\hline$M_{\mathrm{r}}$ & 511.11 \\
\hline Crystal system, space group & Monoclinic, $C 2 / c$ \\
\hline Temperature $(\mathrm{K})$ & 293 \\
\hline$a, b, c(\AA)$ & $\begin{array}{l}10.8154(9), 9.3243(7) \\
13.3313(11)\end{array}$ \\
\hline$\beta\left({ }^{\circ}\right)$ & $106.648(6)$ \\
\hline$V\left(\AA^{3}\right)$ & $1288.05(18)$ \\
\hline$Z$ & 4 \\
\hline Radiation type & Mo $K \alpha$ \\
\hline$\mu\left(\mathrm{mm}^{-1}\right)$ & 13.21 \\
\hline Crystal size (mm) & $0.24 \times 0.15 \times 0.12$ \\
\hline \multicolumn{2}{|l|}{ Data collection } \\
\hline Diffractometer & Stoe $I P D S 2 \mathrm{~T}$ \\
\hline Absorption correction & $\begin{array}{l}\text { Integration }(X-R E D 32 \text { and } \\
\quad X-S H A P E ; \text { Stoe \& Cie, } 2009)\end{array}$ \\
\hline$T_{\min }, T_{\max }$ & $0.103,0.344$ \\
\hline $\begin{array}{l}\text { No. of measured, independent and } \\
\text { observed }[I>2 \sigma(I)] \text { reflections }\end{array}$ & $14009,2710,2298$ \\
\hline$R_{\text {int }}$ & 0.043 \\
\hline$(\sin \theta / \lambda)_{\max }\left(\AA^{-1}\right)$ & 0.798 \\
\hline \multicolumn{2}{|l|}{ Refinement } \\
\hline$R\left[F^{2}>2 \sigma\left(F^{2}\right)\right], w R\left(F^{2}\right), S$ & $0.024,0.053,1.08$ \\
\hline No. of reflections & 2710 \\
\hline No. of parameters & 70 \\
\hline$\Delta \rho_{\max }, \Delta \rho_{\min }\left(\mathrm{e} \AA^{-3}\right)$ & $1.15,-0.75$ \\
\hline
\end{tabular}

Computer programs: $X$-AREA (Stoe \& Cie, 2011), $X$-RED (Stoe \& Cie, 2009), SHELXT2014 (Sheldrick, 2015a), SHELXL2014 (Sheldrick, 2015b), DIAMOND (Brandenburg, 2015) and publCIF (Westrip, 2010).

1952). The positions of the $\mathrm{C}$ and $\mathrm{N}$ atoms were located from a difference-Fourier map.

\section{Acknowledgements}

FK thanks the DFG for his Heisenberg professorship, Dr Harms for X-ray measurement time and Julia Hassler for the sample preparation.

\section{References}

Altermatt, D., Arend, H., Gramlich, V., Niggli, A. \& Petter, W. (1984). Acta Cryst. B40, 347-350.

Bagautdinov, B. S. \& Brown, I. D. (2000). J. Phys. Condens. Matter, 12, 8111-8125.

Beauchamp, A. L. \& Goutier, D. (1972). Can. J. Chem. 50, 977-981.

Brandenburg, K. (2015). DIAMOND. Crystal Impact GbR, Bonn, Germany.

Dickinson, R. G. (1922). J. Am. Chem. Soc. 44, 744-786.

Gerlach, P. N. \& Powell, B. M. (1986). J. Chem. Phys. 85, 6004-6009.

Hermes, O. (1866). J. Prakt. Chem. 97, 465-482.

Jeffery, J. W. \& Rose, K. M. (1968). Acta Cryst. B24, 653-662.

Klüfers, P., Fuess, H. \& Haussühl, S. (1981). Z. Kristallogr. 156, 255 263.

Larbot, A. \& Beauchamp, A. L. (1973). Rev. Chim. Miner. 10, 465472.

Linde, S. A., Mikhailova, A. Y., Pakhomov, V. I., Kirilenko, V. V. \& Shulga, V. G. (1983). Koord. Khim. 9, 998-999.

Pakhomov, V. I. \& Fedorov, P. M. (1973). Sov. Phys. Crystallogr. 17, 833-836.

Pakhomov, V. I., Fedorova, N. M. \& Ivanova Korfini, I. N. (1978). Sov. J. Coord. Chem. 4, 1356-1357.

Pakhomov, V. I., Goryunov, A. V., Gladkii, V. V., Ivanova Korfini, I. N. \& Kallaev, S. N. (1992b). Russ. J. Inorg. Chem. 37, 731-734.

Pakhomov, V. I., Goryunov, A. V., Ivanova Korfini, I. N., Boguslavskii, A. A. \& Lotfullin, R. S. (1992a). Russ. J. Inorg. Chem. 37, 259-261.

Pinheiro, C. B., Jório, A., Pimenta, M. A. \& Speziali, N. L. (1998). Acta Cryst. B54, 197-203.

Rosenheim, A. \& Cohn, R. (1901). Z. Anorg. Allg. Chem. 27, 270303.

Sheldrick, G. M. (2015a). Acta Cryst. A71, 3-8.

Sheldrick, G. M. (2015b). Acta Cryst. C71, 3-8.

Stoe \& Cie (2009). X-RED32 and X-SHAPE. Stoe \& Cie GmbH, Darmstadt, Germany.

Stoe \& Cie (2011). X-AREA. Stoe \& Cie GmbH, Darmstadt, Germany.

Tramer, A. (1962). J. Chem. Phys. 59, 637-654.

Weil, M. \& Häusler, T. (2014). Acta Cryst. E70, i46.

Westrip, S. P. (2010). J. Appl. Cryst. 43, 920-925.

Zandbergen, H. W., Verschoor, G. C. \& IJdo, D. J. W. (1979). Acta Cryst. B35, 1425-1427.

Zvonkova, Z. V. (1952). Zh. Fiz. Khim. 26, 1798-1803. 


\section{supporting information}

Acta Cryst. (2017). E73, 1073-1075 [https://doi.org/10.1107/S2056989017009148]

\section{Redetermination of the crystal structure of $\mathrm{K}_{2} \mathrm{Hg}(\mathrm{SCN})_{4}$}

\section{Jascha Bandemehr, Matthias Conrad and Florian Kraus}

Computing details

Data collection: $X$-AREA (Stoe \& Cie, 2011); cell refinement: X-AREA (Stoe \& Cie, 2011); data reduction: X-RED (Stoe \& Cie, 2009); program(s) used to solve structure: SHELXT2014 (Sheldrick, 2015a); program(s) used to refine structure: SHELXL2014 (Sheldrick, 2015b); molecular graphics: DIAMOND (Brandenburg, 2015); software used to prepare material for publication: publCIF (Westrip, 2010).

Dipotassium tetrathiocyanatomercurate(II)

Crystal data

$\mathrm{K}_{2} \mathrm{Hg}(\mathrm{SCN})_{4}$

$M_{r}=511.11$

Monoclinic, $C 2 / c$

$a=10.8154(9) \AA$

$b=9.3243(7) \AA$

$c=13.3313(11) \AA$

$\beta=106.648(6)^{\circ}$

$V=1288.05(18) \AA^{3}$

$Z=4$

Data collection

Stoe IPDS 2T diffractometer

Radiation source: sealed X-ray tube, 12 x 0.4 $\mathrm{mm}$ long-fine focus

Plane graphite monochromator

Detector resolution: 6.67 pixels $\mathrm{mm}^{-1}$

rotation method scans

Absorption correction: integration

(X-RED32 and X-SHAPE; Stoe \& Cie, 2009)

\section{Refinement}

Refinement on $F^{2}$

Least-squares matrix: full

$R\left[F^{2}>2 \sigma\left(F^{2}\right)\right]=0.024$

$w R\left(F^{2}\right)=0.053$

$S=1.08$

2710 reflections

70 parameters

0 restraints

Primary atom site location: other

Secondary atom site location: other
$F(000)=936$

$D_{\mathrm{x}}=2.636 \mathrm{Mg} \mathrm{m}^{-3}$

Mo $K \alpha$ radiation, $\lambda=0.71073 \AA$

Cell parameters from 25154 reflections

$\theta=2.9-35.0^{\circ}$

$\mu=13.21 \mathrm{~mm}^{-1}$

$T=293 \mathrm{~K}$

Block, colourless

$0.24 \times 0.15 \times 0.12 \mathrm{~mm}$

$T_{\min }=0.103, T_{\max }=0.344$

14009 measured reflections

2710 independent reflections

2298 reflections with $I>2 \sigma(I)$

$R_{\text {int }}=0.043$

$\theta_{\text {max }}=34.6^{\circ}, \theta_{\min }=2.9^{\circ}$

$h=-17 \rightarrow 17$

$k=-14 \rightarrow 14$

$l=-21 \rightarrow 21$

$w=1 /\left[\sigma^{2}\left(F_{\mathrm{o}}^{2}\right)+(0.022 P)^{2}+1.7 P\right]$ where $P=\left(F_{\mathrm{o}}^{2}+2 F_{\mathrm{c}}^{2}\right) / 3$

$(\Delta / \sigma)_{\max }=0.001$

$\Delta \rho_{\max }=1.15 \mathrm{e} \AA^{-3}$

$\Delta \rho_{\min }=-0.75$ e $\AA^{-3}$

Extinction correction: SHELXL2014 (Sheldrick, 2015b), $\mathrm{Fc}^{*}=\mathrm{kFc}\left[1+0.001 \mathrm{xFc}^{2} \lambda^{3} / \sin (2 \theta)\right]^{-1 / 4}$

Extinction coefficient: 0.0086 (2) 


\section{Special details}

Geometry. All esds (except the esd in the dihedral angle between two 1.s. planes) are estimated using the full covariance matrix. The cell esds are taken into account individually in the estimation of esds in distances, angles and torsion angles; correlations between esds in cell parameters are only used when they are defined by crystal symmetry. An approximate (isotropic) treatment of cell esds is used for estimating esds involving l.s. planes.

Fractional atomic coordinates and isotropic or equivalent isotropic displacement parameters $\left(\AA^{2}\right)$

\begin{tabular}{lllll}
\hline & $x$ & $y$ & $z$ & $U_{\text {iso }} * / U_{\text {eq }}$ \\
\hline $\mathrm{Hg}$ & 0.0000 & $0.52099(2)$ & 0.2500 & $0.03803(7)$ \\
$\mathrm{K}$ & $0.16185(7)$ & $1.04695(8)$ & $0.43195(7)$ & $0.04817(17)$ \\
$\mathrm{S} 1$ & $0.10639(7)$ & $0.68302(8)$ & $0.40531(6)$ & $0.03720(14)$ \\
$\mathrm{S} 2$ & $0.18135(7)$ & $0.36527(10)$ & $0.22498(7)$ & $0.04701(18)$ \\
$\mathrm{C} 1$ & $-0.0130(3)$ & $0.6793(3)$ & $0.4611(2)$ & $0.0332(5)$ \\
$\mathrm{C} 2$ & $0.3046(3)$ & $0.4505(3)$ & $0.3055(3)$ & $0.0387(6)$ \\
$\mathrm{N} 1$ & $-0.0940(3)$ & $0.6801(3)$ & $0.5013(3)$ & $0.0462(6)$ \\
$\mathrm{N} 2$ & $0.3926(3)$ & $0.5077(4)$ & $0.3607(4)$ & $0.0615(9)$ \\
\hline
\end{tabular}

Atomic displacement parameters $\left(\AA^{2}\right)$

\begin{tabular}{lllllll}
\hline & $U^{11}$ & $U^{22}$ & $U^{33}$ & $U^{12}$ & $U^{13}$ & $U^{23}$ \\
\hline $\mathrm{Hg}$ & $0.02612(7)$ & $0.05275(11)$ & $0.03471(8)$ & 0.000 & $0.00789(5)$ & 0.000 \\
$\mathrm{~K}$ & $0.0336(3)$ & $0.0491(4)$ & $0.0637(4)$ & $-0.0053(3)$ & $0.0169(3)$ & $-0.0164(3)$ \\
$\mathrm{S} 1$ & $0.0294(3)$ & $0.0399(3)$ & $0.0426(3)$ & $-0.0077(2)$ & $0.0107(2)$ & $-0.0070(3)$ \\
$\mathrm{S} 2$ & $0.0350(3)$ & $0.0567(5)$ & $0.0486(4)$ & $0.0053(3)$ & $0.0108(3)$ & $-0.0162(3)$ \\
$\mathrm{C} 1$ & $0.0307(11)$ & $0.0271(11)$ & $0.0399(12)$ & $-0.0003(9)$ & $0.0070(9)$ & $-0.0026(9)$ \\
$\mathrm{C} 2$ & $0.0304(12)$ & $0.0385(15)$ & $0.0475(14)$ & $0.0066(10)$ & $0.0115(10)$ & $0.0066(11)$ \\
$\mathrm{N} 1$ & $0.0408(13)$ & $0.0437(14)$ & $0.0586(17)$ & $-0.0011(11)$ & $0.0215(12)$ & $-0.0039(12)$ \\
$\mathrm{N} 2$ & $0.0346(14)$ & $0.0530(19)$ & $0.087(3)$ & $0.0011(12)$ & $0.0023(14)$ & $0.0011(16)$ \\
\hline
\end{tabular}

Geometric parameters $\left(\AA,{ }^{\circ}\right)$

\begin{tabular}{llll}
\hline $\mathrm{Hg}-\mathrm{S} 2$ & $2.5380(8)$ & $\mathrm{K}-\mathrm{K}^{\mathrm{iv}}$ & $4.4669(15)$ \\
$\mathrm{Hg}-\mathrm{S} 2^{\mathrm{i}}$ & $2.5380(8)$ & $\mathrm{S} 1-\mathrm{C} 1$ & $1.665(3)$ \\
$\mathrm{Hg}-\mathrm{S} 1^{\mathrm{i}}$ & $2.5550(7)$ & $\mathrm{S} 1-\mathrm{K}^{\mathrm{v}}$ & $3.5316(12)$ \\
$\mathrm{Hg}-\mathrm{S} 1$ & $2.5551(7)$ & $\mathrm{S} 2-\mathrm{C} 2$ & $1.656(3)$ \\
$\mathrm{K}-\mathrm{N} 2^{\mathrm{ii}}$ & $2.816(4)$ & $\mathrm{S} 2-\mathrm{K}^{\mathrm{viii}}$ & $3.4865(12)$ \\
$\mathrm{K}-\mathrm{N} 1^{\mathrm{iii}}$ & $2.823(3)$ & $\mathrm{C} 1-\mathrm{N} 1$ & $1.152(4)$ \\
$\mathrm{K}-\mathrm{N} 1^{\text {iv }}$ & $2.860(3)$ & $\mathrm{C} 1-\mathrm{K}^{\mathrm{iv}}$ & $3.529(3)$ \\
$\mathrm{K}-\mathrm{N} 2^{\mathrm{v}}$ & $3.031(5)$ & $\mathrm{C} 2-\mathrm{N} 2$ & $1.153(5)$ \\
$\mathrm{K}-\mathrm{C} 2^{\mathrm{vi}}$ & $3.408(3)$ & $\mathrm{C} 2-\mathrm{K}^{\mathrm{viii}}$ & $3.408(3)$ \\
$\mathrm{K}-\mathrm{C} 2^{\mathrm{v}}$ & $3.414(3)$ & $\mathrm{C} 2-\mathrm{K}^{\mathrm{v}}$ & $3.414(3)$ \\
$\mathrm{K}-\mathrm{S} 1$ & $3.4466(11)$ & $\mathrm{N} 1-\mathrm{K}^{\mathrm{ix}}$ & $2.823(3)$ \\
$\mathrm{K}-\mathrm{S} 2^{\mathrm{vi}}$ & $3.4865(12)$ & $\mathrm{N} 1-\mathrm{K}^{\mathrm{iv}}$ & $2.860(3)$ \\
$\mathrm{K}-\mathrm{S} 1^{\mathrm{v}}$ & $3.5315(12)$ & $\mathrm{N} 2-\mathrm{K}^{\mathrm{x}}$ & $2.816(4)$ \\
$\mathrm{K}-\mathrm{C} 1^{\text {iv }}$ & $3.529(3)$ & $\mathrm{N} 2-\mathrm{K}^{\mathrm{v}}$ & $3.031(5)$ \\
$\mathrm{K}-\mathrm{K}^{\mathrm{vii}}$ & $4.3913(14)$ & &
\end{tabular}




\begin{tabular}{|c|c|c|c|}
\hline $\mathrm{S} 2-\mathrm{Hg}-\mathrm{S} 2^{\mathrm{i}}$ & $110.21(4)$ & $\mathrm{S} 1-\mathrm{K}-\mathrm{C} 1^{\mathrm{iv}}$ & $131.89(5)$ \\
\hline $\mathrm{S} 2-\mathrm{Hg}-\mathrm{S} 1^{\mathrm{i}}$ & $114.67(3)$ & $\mathrm{S} 2^{\mathrm{vi}}-\mathrm{K}-\mathrm{C} 1^{\mathrm{iv}}$ & $161.89(6)$ \\
\hline $\mathrm{S} 22^{\mathrm{i}}-\mathrm{Hg}-\mathrm{S} 1^{\mathrm{i}}$ & $105.02(2)$ & $\mathrm{S} 1^{\mathrm{v}}-\mathrm{K}-\mathrm{C} 1^{\mathrm{iv}}$ & $121.11(5)$ \\
\hline $\mathrm{S} 2-\mathrm{Hg}-\mathrm{S} 1$ & $105.02(2)$ & $\mathrm{N} 2^{\mathrm{ii}}-\mathrm{K}-\mathrm{K}^{\mathrm{vii}}$ & $122.43(8)$ \\
\hline $\mathrm{S} 2 \mathrm{i}-\mathrm{Hg}-\mathrm{S} 1$ & 114.67 (3) & $\mathrm{N} 1^{\mathrm{iii}-\mathrm{K}}-\mathrm{K}^{\mathrm{vii}}$ & $39.71(6)$ \\
\hline $\mathrm{S} 1 \mathrm{i}-\mathrm{Hg}-\mathrm{S} 1$ & $107.50(4)$ & $\mathrm{N} 1^{\mathrm{iv}}-\mathrm{K}-\mathrm{K}^{\mathrm{vii}}$ & $39.09(6)$ \\
\hline $\mathrm{N} 2^{\mathrm{ii}}-\mathrm{K}-\mathrm{N} 1^{\mathrm{iii}}$ & $161.33(10)$ & $\mathrm{N} 2^{\mathrm{v}}-\mathrm{K}-\mathrm{K}^{\mathrm{vii}}$ & $86.73(7)$ \\
\hline $\mathrm{N} 2^{\mathrm{ii}-\mathrm{K}}-\mathrm{N} 1^{\mathrm{iv}}$ & $83.58(10)$ & $\mathrm{C} 2^{\mathrm{vi}}-\mathrm{K}-\mathrm{K}^{\mathrm{vii}}$ & $117.52(6)$ \\
\hline $\mathrm{N} 1^{\mathrm{iii}-\mathrm{K}}-\mathrm{N} 1^{\mathrm{iv}}$ & $78.80(9)$ & $\mathrm{C} 2^{\mathrm{v}}-\mathrm{K}-\mathrm{K}^{\mathrm{vii}}$ & $70.39(6)$ \\
\hline 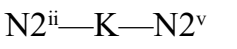 & 80.42 (13) & $\mathrm{S} 1-\mathrm{K}-\mathrm{K}^{\mathrm{vii}}$ & $159.02(4)$ \\
\hline $\mathrm{N} 1^{\mathrm{iii}}-\mathrm{K}-\mathrm{N} 2^{\mathrm{v}}$ & $100.55(9)$ & $\mathrm{S} 2^{\mathrm{vi}}-\mathrm{K}-\mathrm{K}^{\mathrm{vii}}$ & $116.01(3)$ \\
\hline $\mathrm{N} 1^{\mathrm{iv}}-\mathrm{K}-\mathrm{N} 2^{\mathrm{v}}$ & $74.44(9)$ & $\mathrm{S} 1^{\mathrm{v}}-\mathrm{K}-\mathrm{K}^{\mathrm{vii}}$ & $97.01(3)$ \\
\hline $\mathrm{N} 2^{\mathrm{ii}-\mathrm{K}}-\mathrm{C} 2^{\mathrm{vi}}$ & 91.55 (12) & $\mathrm{C} 1^{\mathrm{iv}}-\mathrm{K}-\mathrm{K}^{\mathrm{vii}}$ & $53.40(5)$ \\
\hline $\mathrm{N} 1^{\mathrm{iii}}-\mathrm{K}-\mathrm{C} 2^{\mathrm{vi}}$ & $94.70(8)$ & $\mathrm{N} 2^{\mathrm{ii}}-\mathrm{K}-\mathrm{K}^{\mathrm{iv}}$ & $41.99(10)$ \\
\hline $\mathrm{N} 1^{\mathrm{iv}}-\mathrm{K}-\mathrm{C} 2^{\mathrm{vi}}$ & $128.94(9)$ & $\mathrm{N} 1^{\mathrm{iii}}-\mathrm{K}-\mathrm{K}^{\mathrm{iv}}$ & $136.17(7)$ \\
\hline $\mathrm{N} 2^{\mathrm{v}}-\mathrm{K}-\mathrm{C} 2^{\mathrm{vi}}$ & 154.57 (9) & $\mathrm{N} 1^{\mathrm{iv}}-\mathrm{K}-\mathrm{K}^{\mathrm{iv}}$ & $75.38(6)$ \\
\hline $\mathrm{N} 2^{\mathrm{ii}}-\mathrm{K}-\mathrm{C} 2^{\mathrm{v}}$ & $98.21(12)$ & $\mathrm{N} 2^{\mathrm{v}}-\mathrm{K}-\mathrm{K}^{\mathrm{iv}}$ & $38.43(7)$ \\
\hline $\mathrm{N} 1^{\mathrm{iii}-\mathrm{K}}-\mathrm{C} 2^{\mathrm{v}}$ & $81.16(8)$ & $\mathrm{C} 2^{\mathrm{vi}}-\mathrm{K}-\mathrm{K}^{\mathrm{iv}}$ & $129.03(5)$ \\
\hline $\mathrm{N} 1^{\mathrm{iv}}-\mathrm{K}-\mathrm{C} 2^{\mathrm{v}}$ & $68.68(8)$ & $\mathrm{C} 2^{\mathrm{v}}-\mathrm{K}-\mathrm{K}^{\mathrm{iv}}$ & $56.66(5)$ \\
\hline $\mathrm{N} 2^{\mathrm{v}}-\mathrm{K}-\mathrm{C} 2^{\mathrm{v}}$ & $19.47(8)$ & $\mathrm{S} 1-\mathrm{K}-\mathrm{K}^{\mathrm{iv}}$ & $73.46(2)$ \\
\hline $\mathrm{C} 2^{\mathrm{vi}}-\mathrm{K}-\mathrm{C} 2^{\mathrm{v}}$ & $161.02(7)$ & $\mathrm{S} 2^{\mathrm{vi}}-\mathrm{K}-\mathrm{K}^{\mathrm{iv}}$ & $136.14(3)$ \\
\hline $\mathrm{N} 2{ }^{\mathrm{ii}}-\mathrm{K}-\mathrm{S} 1$ & $72.82(7)$ & $\mathrm{S} 1^{\mathrm{v}}-\mathrm{K}-\mathrm{K}^{\mathrm{iv}}$ & $97.61(3)$ \\
\hline $\mathrm{N} 1^{\mathrm{iii}}-\mathrm{K}-\mathrm{S} 1$ & $125.84(7)$ & $\mathrm{C} 1^{\mathrm{iv}}-\mathrm{K}-\mathrm{K}^{\mathrm{iv}}$ & $58.43(5)$ \\
\hline $\mathrm{N} 1^{\text {iv }}-\mathrm{K}-\mathrm{S} 1$ & $148.83(6)$ & $\mathrm{K}^{\mathrm{vii}}-\mathrm{K}-\mathrm{K}^{\mathrm{iv}}$ & $107.42(3)$ \\
\hline $\mathrm{N} 2^{\mathrm{v}}-\mathrm{K}-\mathrm{S} 1$ & $81.66(7)$ & $\mathrm{C} 1-\mathrm{S} 1-\mathrm{Hg}$ & $97.06(10)$ \\
\hline $\mathrm{C} 2^{\mathrm{vi}-\mathrm{K}}-\mathrm{S} 1$ & $72.91(5)$ & $\mathrm{C} 1-\mathrm{S} 1-\mathrm{K}$ & $96.25(9)$ \\
\hline $\mathrm{C} 2^{\mathrm{v}}-\mathrm{K}-\mathrm{S} 1$ & $94.40(6)$ & $\mathrm{Hg}-\mathrm{S} 1-\mathrm{K}$ & $133.66(3)$ \\
\hline $\mathrm{N} 2^{\mathrm{ii}}-\mathrm{K}-\mathrm{S} 2^{\mathrm{vi}}$ & $111.59(10)$ & $\mathrm{C} 1-\mathrm{S} 1-\mathrm{K}^{\mathrm{v}}$ & $102.56(10)$ \\
\hline $\mathrm{N} 1^{\mathrm{iii}}-\mathrm{K}-\mathrm{S} 2^{\mathrm{vi}}$ & $80.81(6)$ & $\mathrm{Hg}-\mathrm{S} 1-\mathrm{K}^{\mathrm{v}}$ & $102.38(3)$ \\
\hline $\mathrm{N} 1^{\mathrm{iv}}-\mathrm{K}-\mathrm{S} 2^{\mathrm{vi}}$ & $146.21(6)$ & $\mathrm{K}-\mathrm{S} 1-\mathrm{K}^{\mathrm{v}}$ & $117.55(2)$ \\
\hline $\mathrm{N} 2^{\mathrm{v}}-\mathrm{K}-\mathrm{S} 2^{\mathrm{vi}}$ & $136.13(7)$ & $\mathrm{C} 2-\mathrm{S} 2-\mathrm{Hg}$ & $98.59(10)$ \\
\hline $\mathrm{C} 2^{\mathrm{vi}}-\mathrm{K}-\mathrm{S} 2^{\mathrm{vi}}$ & $27.76(5)$ & $\mathrm{C} 2-\mathrm{S} 2-\mathrm{K}^{\mathrm{viii}}$ & $73.50(11)$ \\
\hline $\mathrm{C} 2^{\mathrm{v}}-\mathrm{K}-\mathrm{S} 2^{\mathrm{vi}}$ & $133.75(5)$ & $\mathrm{Hg}-\mathrm{S} 2-\mathrm{K}^{\mathrm{viii}}$ & $109.15(3)$ \\
\hline $\mathrm{S} 1-\mathrm{K}-\mathrm{S} 2^{\mathrm{vi}}$ & $63.89(2)$ & $\mathrm{N} 1-\mathrm{C} 1-\mathrm{S} 1$ & $178.0(3)$ \\
\hline $\mathrm{N} 2^{\mathrm{ii}}-\mathrm{K}-\mathrm{S} 1^{\mathrm{v}}$ & $127.71(8)$ & $\mathrm{N} 1-\mathrm{C} 1-\mathrm{K}^{\mathrm{iv}}$ & $46.43(18)$ \\
\hline $\mathrm{N} 1^{\mathrm{iii}}-\mathrm{K}-\mathrm{S} 1^{\mathrm{v}}$ & 68.47 (7) & $\mathrm{S} 1-\mathrm{C} 1-\mathrm{K}^{\mathrm{iv}}$ & $131.85(12)$ \\
\hline $\mathrm{N} 1^{\mathrm{iv}}-\mathrm{K}-\mathrm{S} 1^{\mathrm{v}}$ & $123.33(7)$ & $\mathrm{N} 2-\mathrm{C} 2-\mathrm{S} 2$ & $178.2(3)$ \\
\hline $\mathrm{N} 2^{\mathrm{v}}-\mathrm{K}-\mathrm{S} 1^{\mathrm{v}}$ & $68.09(7)$ & $\mathrm{N} 2-\mathrm{C} 2-\mathrm{K}^{\mathrm{viii}}$ & $100.5(3)$ \\
\hline $\mathrm{C} 2^{\mathrm{vi}}-\mathrm{K}-\mathrm{S} 1^{\mathrm{v}}$ & $99.48(6)$ & $\mathrm{S} 2-\mathrm{C} 2-\mathrm{K}^{\mathrm{viii}}$ & $78.74(12)$ \\
\hline $\mathrm{C} 2^{\mathrm{v}}-\mathrm{K}-\mathrm{S} 1^{\mathrm{v}}$ & $61.72(5)$ & $\mathrm{N} 2-\mathrm{C} 2-\mathrm{K}^{\mathrm{v}}$ & $61.1(3)$ \\
\hline $\mathrm{S} 1-\mathrm{K}-\mathrm{S} 1^{\mathrm{v}}$ & $62.45(2)$ & $\mathrm{S} 2-\mathrm{C} 2-\mathrm{K}^{\mathrm{v}}$ & $119.82(14)$ \\
\hline $\mathrm{S} 2^{\mathrm{vi}}-\mathrm{K}-\mathrm{S} 1^{\mathrm{v}}$ & $72.06(2)$ & $\mathrm{K}^{\mathrm{viii}}-\mathrm{C} 2-\mathrm{K}^{\mathrm{v}}$ & $160.38(10)$ \\
\hline $\mathrm{N} 2^{\mathrm{ii}-\mathrm{K}}-\mathrm{C} 1^{\mathrm{iv}}$ & $71.53(9)$ & $\mathrm{C} 1-\mathrm{N} 1-\mathrm{K}^{\mathrm{ix}}$ & $127.6(2)$ \\
\hline $\mathrm{N} 1^{\mathrm{iii}-\mathrm{K}}-\mathrm{C} 1^{\mathrm{iv}}$ & $92.39(7)$ & $\mathrm{C} 1-\mathrm{N} 1-\mathrm{K}^{\mathrm{iv}}$ & $116.6(2)$ \\
\hline $\mathrm{N} 1^{\mathrm{iv}}-\mathrm{K}-\mathrm{C} 1^{\mathrm{iv}}$ & $16.96(7)$ & $\mathrm{K}^{\mathrm{ix}}-\mathrm{N} 1-\mathrm{K}^{\mathrm{iv}}$ & $101.20(9)$ \\
\hline $\mathrm{N} 2^{\mathrm{v}}-\mathrm{K}-\mathrm{C} 1^{\mathrm{iv}}$ & $61.48(8)$ & $\mathrm{C} 2-\mathrm{N} 2-\mathrm{K}^{\mathrm{x}}$ & $149.3(3)$ \\
\hline
\end{tabular}




\begin{tabular}{llll}
$\mathrm{C} 2^{\mathrm{vi}}-\mathrm{K}-\mathrm{C}^{\mathrm{iv}}$ & $138.43(8)$ & $\mathrm{C} 2-\mathrm{N} 2-\mathrm{K}^{\mathrm{v}}$ & $99.4(3)$ \\
$\mathrm{C} 2^{\mathrm{v}}-\mathrm{K}-\mathrm{C} 1^{\mathrm{iv}}$ & $60.50(7)$ & $\mathrm{K}^{\mathrm{x}}-\mathrm{N} 2-\mathrm{K}^{\mathrm{v}}$ & $99.58(13)$ \\
\hline
\end{tabular}

Symmetry codes: (i) $-x, y,-z+1 / 2$; (ii) $x-1 / 2, y+1 / 2, z$; (iii) $x+1 / 2, y+1 / 2, z$; (iv) $-x,-y+2,-z+1$; (v) $-x+1 / 2,-y+3 / 2,-z+1$; (vi) $-x+1 / 2, y+1 / 2,-z+1 / 2$; (vii) $-x+1 / 2,-y+5 / 2,-z+1$; (viii) $-x+1 / 2, y-1 / 2,-z+1 / 2$; (ix) $x-1 / 2, y-1 / 2, z$; (x) $x+1 / 2, y-1 / 2, z$. 